\section{Propuesta de reformas a los prestadores públicos de servicios médicos en Chile: "fortaleciendo la opción pública"}

\author{
MARCOS VERGARA I.
}

\section{A reform proposal to strengthen public health care}

Currently, there is no discussion on the need to improve and strengthen the institutional health care modality of FONASA (MAI), the health care system used by the public services net and by most of the population, despite the widely known and long lasting problems such as waiting lists, hospital debt with suppliers, lack of specialists and increasing services purchase transference to the private sector, etc. In a dichotomous sectorial context, such as the one of health's social security in Chile (the state on one side and the market on the other), points of view are polarized and stances tend to seek refuge within themselves. As a consequence, to protect the public solution is commonly associated with protecting the "status quo", creating an environment that is reluctant to change. The author proposes a solution based on three basic core ideas, which, if proven effective, can strengthen each other if combined properly. These are: network financing management, governance of health care services in MAI and investments and human resources in networked self-managed institutions. The proposal of these core ideas was done introducing a reality testing that minimizes the politic complexity of their implementation.

(Rev Med Chile 2015; 143: 237-243)

Key words (MeSH): Clinical governance; Health manpower; Investments; Health services administration; Public sector.

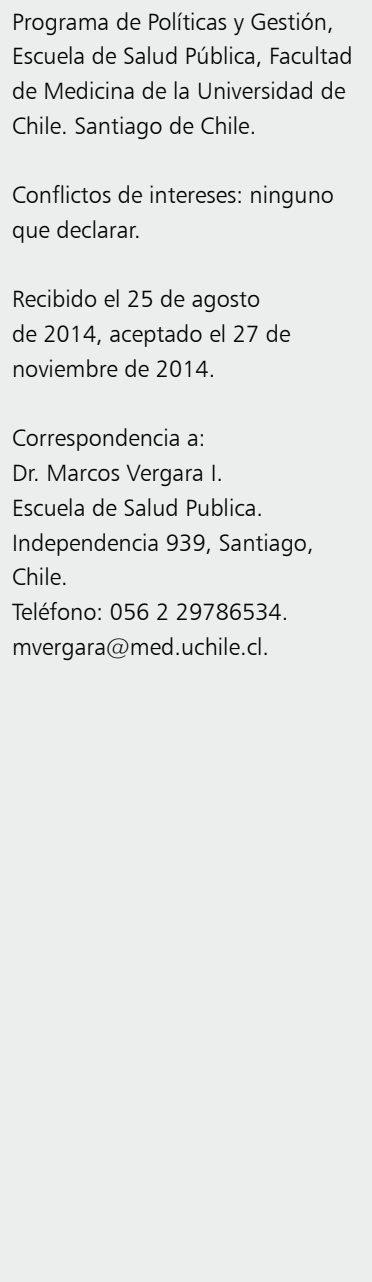

$\mathrm{P}$ odrá resultar extraña a algunos la exploración de este tema de la provisión pública de servicios, cuando todos los "cartuchos" intelectuales del sector han estado destinados a definir el destino de las Isapre (Instituciones de Salud Previsional). De hecho, apremiado por el Tribunal Constitucional, el gobierno anterior formó dos comisiones sucesivas para ver la materia ${ }^{1,2}$ y el actual gobierno formó la suya propia. Sin embargo, si escapamos del manto de autocomplacencia que a veces tejemos sobre el desempeño de la provisión pública y de la irresistible tentación de proteger el status $q u o^{3}$, es necesario plantearse cómo es que la provisión pública de servicios en Chile, donde se atiende la gran mayoría de nuestros compatriotas, podría resultar efectivamente fortalecida. Y lo que parece necesario plantear para ello, como en cualquier organización que está atenta a las necesidades de sus usuarios y a su entorno relevante, es precisamente la realización de algunos cambios ${ }^{4}$, en particular cuando, como en el caso chileno, los hospitales públicos compiten fuertemente con los prestadores privados para obtener recursos especializados en tiempos de escasez de los mismos (anestesistas, utiólogos y otros).

Lo anterior no está sólo alentado por la idea de poner el foco en lo sustantivo, como algunos expertos dicen que no se hizo desde la partida en la reforma educacional -primero, la educación pública-, sino porque, además, si estuviera en perspectiva una solución financiera de fondo único en Fonasa (Fondo Nacional de Salud) y las 
Isapres fueran remitidas al mercado de los seguros complementarios, las opciones que habrán de ofrecerse a los beneficiarios antiguos y nuevos de la seguridad social en un escenario como éste, han de ser de calidad ${ }^{5}$. Una de esas opciones es la modalidad de atención institucional (MAI) que se brinda en la red pública, sobre la que recae ahora el peso de la prueba: mostrar que una solución "cerrada" puede ser del todo satisfactoria para quienes la usan y a la vez costo-efectiva ${ }^{6}$.

\section{La enfermedad y sus causas}

La producción hospitalaria pública de servicios a beneficiarios de Fonasa (egresos y días-cama) ha permanecido constante en la última década, mientras se reproduce la deuda con proveedores, las listas de espera no GES demandan esfuerzos especiales para su reducción con magros resultados-pedaleamos la bicicleta-y las atenciones a los citados beneficiarios crece en las clínicas privadas, espontáneamente o por desbordes de la capacidad instalada. En la actualidad es posible reconocer problemas importantes en esta modalidad de atención (MAI), que representa Fonasa como cliente principal, cuya solución configuraría una agenda de cambios orientada a fortalecer la opción pública de prestación de servicios. Puesto que no se trata de hacer una lista de problemas que en cualquier organización podría ser interminable, nos concentraremos en los grandes problemas de la prestación pública de servicios de salud; es decir en los problemas gruesos o más bien estructurales, que son los siguientes:

1. Fonasa no financia la gestión en RED y se preocupa directamente y sin resultados del control financiero de los servicios de salud -hospitales-, lo que es impropio de un asegurador. Los incentivos de la compra actual pervierten la puesta en práctica de la ventaja comparativa principal de la oferta pública: la provisión a través de la gestión de una red asistencial de resolutividad creciente, amplia en la base (cobertura) y profunda en la cúspide (alta resolutividad) que, desde una perspectiva de salud pública ortodoxa acompaña muy bien a los denominados niveles de prevención de la historia natural de la enfermedad.

2. El sistema de gobierno hospitalario actual-más ampliamente, quizás podríamos decir "su orgánica institucionalizada"8- es frágil, con dependencia unipersonal desde los directores de Servicios de Salud y, habida cuenta de la dependencia que los directores de Servicios de Salud tienen a su vez del Subsecretario de Redes Asistenciales, finalmente el gobierno hospitalario está en manos del Ministerio de Salud ${ }^{9}$, que es el ente rector/regulador del sector y garante de la salud pública para todos los ciudadanos por igual, confundiéndose de este modo las funciones que cabe cumplir a las distintas agencias ${ }^{10}$.

De acuerdo a lo antes señalado, el sistema de toma de decisiones a nivel hospitalario se aleja del lugar en que tomar estas decisiones tiene más sentido ${ }^{11}$, la calidad de las decisiones empeora y los riesgos asociados a las mismas son completamente transferidos a la autoridad superior, cuyo tiempo para la toma de decisiones racionales es más escaso frente a la demanda por ellas que proviene desde los hospitales ${ }^{12}$. En este esquema, el Ministro de Salud termina siendo el responsable de cada cosa que suceda en cualquier hospital de la red asistencial, lo que trae consigo, además, un problema de "accountability".

3. El marco jurídico-administrativo es restrictivo para una empresa prestadora de servicios como también es en realidad un hospital público, producto de lo cual: i) se invierte poco y no se invierte oportunamente, lo que genera quiebres cotidianos en la producción; ii) el capital se deteriora rápidamente por insuficiencia de recursos destinados a mantención; iii) han proliferado a lo largo del tiempo soluciones diversas para expandir en la práctica la dotación de personal (corporaciones de ayuda, fundaciones, compra de servicios de personal clínico) y; iv) los especialistas médicos más escasos de los hospitales se han privatizado en la práctica, agrupados en sociedades lucrativas para la venta de servicios a los hospitales a precios de mercado, los que se financian a través del subtítulo 22 del presupuesto impactando también de este modo en la reproducción de la deuda.

\section{El tratamiento}

Estos tres grandes problemas así presentados y ordenados, permiten organizar un conjunto de soluciones eficaces para intentar resolverlos. Se 
propone, en consecuencia, un abordaje sistémico que conste de una combinación apropiada y potencialmente sinérgica de lo siguiente:

1. Rediseño del sistema de financiamiento actual de la modalidad de atención institucional del Fonasa (MAI) que considera un per cápita en APS y el pago parcial por paquetes de prestaciones (PPV) a los hospitales -ajuste PPI mediante-, a través de los Servicios de Salud.

Se propone un esquema escalonado ${ }^{13}$, donde los recursos se asignen primero globalmente a los Servicios de Salud sobre una base capitada según población a cargo -la suma de las inscripciones en APS- ajustada por variables que aseguren equidad en el acceso, según riesgo de enfermar de la población.

A continuación, el Servicio de Salud asignaría en forma capitada -con los mismos ajustes u otros más sensibles- a APS, pero crearía para la misma un fondo para comprar interconsultas de especialidad controladas por estándar de derivaciones sobre el total de consultas otorgadas, de cuyo margen, cuando exista, la APS podría apropiarse. Los hospitales autogestionados en red (EAR) se financiarían desde los Servicios de Salud sobre una base como la actual, esto es pago por paquetes de prestaciones (PPV) y PPI (este último decreciente durante el tiempo de ajuste), más los fondos provenientes de APS por interconsultas bien resueltas (controles incluidos) y adecuadamente contra-referidas (Figura 1).

Siguiendo a Leavell y Clark, antes citados, lo anterior promovería una conducta anticipativa a la aparición o profundización de los daños en los agentes que participan en los distintos niveles de prevención que componen la red, a través de la resolución oportuna de problemas en el nivel que ha sido dispuesto en la misma para tal efecto, según riesgo, gravedad o complejidad, procurando reducir las complicaciones y la pérdida de años de vida saludables.

En este contexto en que miramos la red y su gestión como ventaja competitiva, superamos la fórmula de que la APS deba aumentar su capacidad resolutiva, como dogmáticamente suele plantearse desde tiempos muy pretéritos (mismo paradigma que nos ha ido moviendo hacia la urgencialización de la asistencia, como una suerte de red paralela a la red electiva). El caso es mirar horizontalmente siguiendo la historia natural de la enfermedad (la denominada continuidad asistencial) y conseguir que la APS sea capaz de controlar apropiadamente los riesgos de enfermar promoviendo la salud y, por ejemplo, mejorando sustantivamente el control del riesgo cardiovascular a ese nivel, entre otros ${ }^{14}$. El incremento o no de la capacidad resolutiva ambulatoria derivará de las particularidades de la red asistencial en que dicha capacidad se inserta $y$,

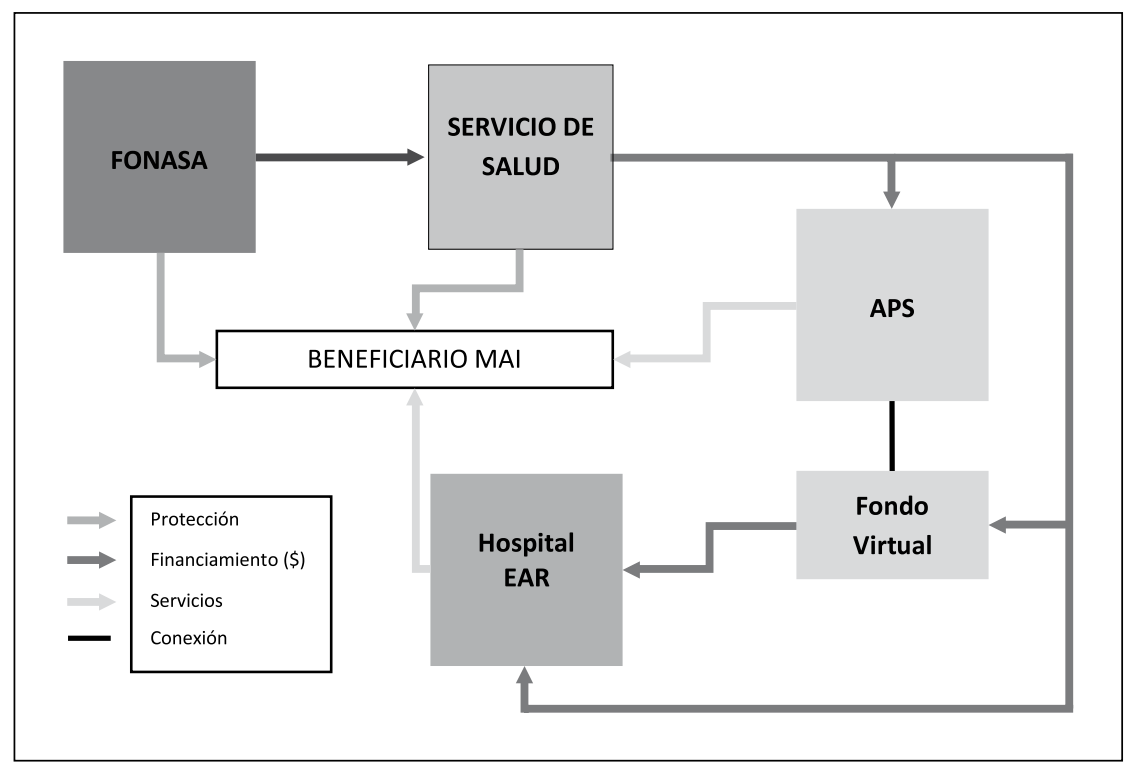

Figura 1. Flujos de protección, financiamiento y servicios en el modelo MAI. 
cuando sea necesario, del propio desarrollo de la medicina, que afectará el desarrollo de la red asistencial como un todo. Es necesario recordar que con el mismo argumento en el pasado reciente se desplegaron alternativas como los denominados Centros de Referencia de Salud (CRS), que representaban nuevas formas de potenciar la capacidad resolutiva ambulatoria con mayores economías de escala, así como los Centros Diagnóstico-Terapéuticos (CDT) en tanto agencias no capturadas por el hospital, ambos tipos desarrollados por el MINSAL a comienzos de los 90 (Proyecto MINSAL/Banco Mundial)) y que a la fecha no han sido evaluados respecto de su concepción, puesta en práctica y propósitos originales.

Sin realizar nuevas modificaciones estructurales en la red (que por lo general ocurren en el largo plazo, inversiones mediante), la propuesta rescata la idea de poner incentivos correctos para la acción, en el entendido que gestionar la red no consiste sólo en gestionar la oferta de servicios (adaptarla a la demanda, lo que es por cierto indispensable), sino también modular la demanda por servicios en perspectiva sanitaria, dado el conocimiento que se tiene de la población a cargo $y$ de sus riesgos de enfermar ${ }^{15}$.

2. La generación de Directorios para hospitales autogestionados en red (EAR) dependientes de la Dirección del Servicio de Salud se perfila como una posibilidad, si bien ésta sería una idea contradictoria con un vínculo del tipo "comprador-prestador" entre el Gestor de Red (Servicio de Salud) y el Hospital, como se ha propuesto en el punto anterior. Ese tipo de vínculo determinaría fuertemente la relación entre Gestor de Red y Hospital. En un escenario con relación "comprador-prestador", ésta debería reducirse a un sólido compromiso de gestión que brindaría la suficiente autonomía al hospital para gestionar los recursos necesarios para generar los "outputs" (servicios, prestaciones, paquetes) de ese compromiso. El "quid" del asunto -el foco, dicho de otro modo- estaría entonces puesto allí y no disperso en un enorme número de indicadores y expresado en variadas formas de dependencia informal, como ocurre en la práctica en la actualidad.

Si prospera la idea de cambiar los mecanismos de financiamiento para la "gestión en red", sólo se requeriría volver a pensar en desconcentrar a la Subsecretaría de Redes del MINSAL e instalar allí el sistema de gobierno, con un modelo del tipo ex SNS (Figura 2) y con un directorio multidisciplinario que minimice los riesgos de las decisiones, evite la captura del sistema por parte de grupos de interés y asegure una buena "accountability" del sistema. Por cierto, haría falta aquí la instalación de un buen brazo armado para garantizar equilibrio financiero y buena productividad en la totalidad de la red, tarea que en la actualidad intenta realizar Fonasa, quien ha gozado de la confianza de la Dirección de Presupuestos para esos efectos. Esto permitiría al Ministerio de Salud concentrarse en las funciones esenciales de salud pública ${ }^{16}$ y a Fonasa actuar como seguro/ comprador, financiando lógicamente per cápita la modalidad de atención institucional (MAI) y crecientemente por paquetes la modalidad de libre elección (MLE).

3. Ahora bien, ante la improbabilidad de contar para los hospitales autogestionados en red (EAR) con un marco jurídico-administrativo distinto al estatuto administrativo, que las organizaciones de trabajadores legítimamente defienden (ver el caso de los establecimientos experimentales y la posición sostenida frente a las concesiones), es necesario incorporar algunas propuestas en materia de Inversión y de Recursos Humanos que sean realizables sin tener que recurrir a la construcción de un nuevo marco jurídico-administrativo o recurrir al de las empresas autónomas del Estado (Codelco/ENAP), que suele ser planteado en los debates acerca de la MAI:

\section{Inversiones}

En Chile, el Estado se hizo cargo muy precozmente de desarrollar una red asistencial pública de salud. El Ministerio de Salud, en tanto dueño de los hospitales y a diferencia de otros ministerios, necesita entonces recursos para realizar, de manera ineludible, cuantiosas y crecientes inversiones para el cumplimiento de las tareas propias de este giro que históricamente el Estado ha asumido en Chile: producción de servicios médicos o clínicos.

Lo anterior es clave en el desarrollo del sector e implica una mayor tasa de inversión anual y una urgente actualización de servicios, estándares y criterios de priorización, tanto como una mo- 


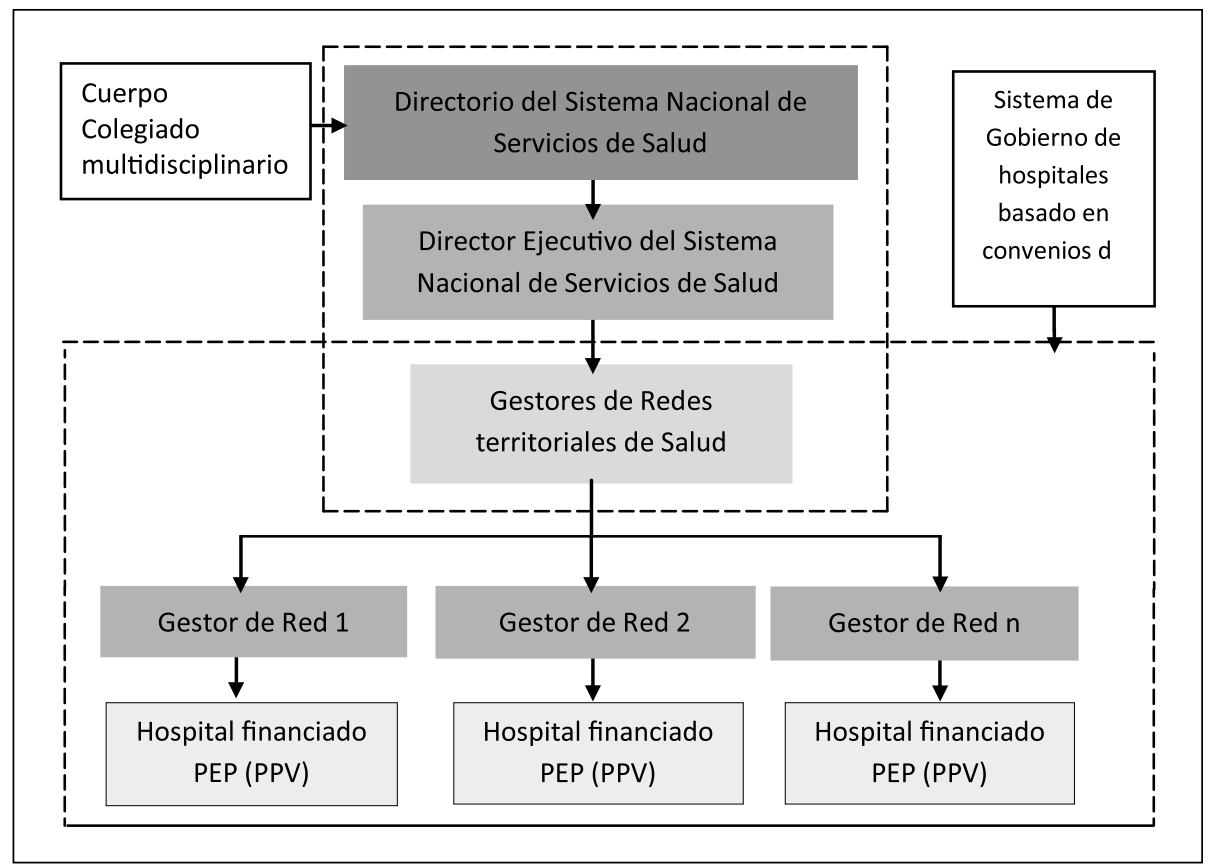

Figura 2. Sistemas de gobierno corporativo desconcentrado. dernización de la formulación y ejecución presupuestaria (entre otros, más subtítulo 29 para las inversiones autónomas de reposición y actualización del capital depreciado en los hospitales EAR). Aquí se arrastra una brecha de enorme magnitud que determina un plan de inversiones de más de 4.000 millones de dólares sólo en los 4 años de este gobierno, lo que ya pone en jaque a la capacidad instalada en el país para lograr materializar tan ambicioso programa.

En paralelo a lo anterior, se requiere realizar el gasto apropiado y suficiente en mantenimiento de edificios y equipos, activos fijos que constituyen el capital del sector, el que se deteriora rápidamente año tras año por recursos insuficientes en la materia y porque los gestores hospitalarios carecen de incentivos para cuidar el capital cuando resulta de inversiones que terceros realizan y que en algún momento tendrán que volver a realizar.

\section{Recursos Humanos}

Se debe realizar la transferencia definitiva de la gestión de Recursos Humanos desde los Servicios de Salud a los Hospitales autogestionados en red (EAR), lo que supone completar ajustes jurídiconormativos que hoy la limitan y la mantienen todavía en manos de los Servicios de Salud.
Es necesario reducir los mecanismos informales de expansión de la dotación de personal (contratación de servicios, uso para esos efectos de las fundaciones y corporaciones de ayuda, etc.) por la pérdida de control sobre el gasto que esto ha venido ocasionando -es indudable que ha ido teniendo creciente impacto en la reproducción de la deuda- y también por el desapego que estos mecanismos producen entre los profesionales y el hospital, entidad que en este tipo de escenario, para los médicos se transforma en una suerte de clínica privada, de la que "entran y salen".

Es preciso distinguir dos fenómenos: primero, la dotación insuficiente, sin que medie escasez de ese tipo de personal en el mercado del trabajo del sector salud. Simplemente, no se cuenta con cargos para completar turnos y actividades de cuidados, lo que ocurre en el caso de enfermeras y técnicos paramédicos. En segundo lugar, el caso de los profesionales escasos, donde se cuenta con los cargos pero no se llenan como consecuencia de que el sector privado puede hasta triplicar el valor hora que ofrece respecto del ofrecido en los hospitales públicos. Aquí está el típico caso de los anestesistas y de los médicos para hacer turnos en unidades de pacientes críticos (UCI, UTI).

En cuanto a la escasez, se vincula con ello el 
fenómeno de la concentración del tiempo médico en las mañanas, de lo que resulta que en las tardes la producción cae a consecuencia de que falta un factor productivo principal, mientras el resto de los factores (personal, equipos e instalaciones) redunda. Pero esto, que puede ser crítico para la reducción de listas de espera, no debe confundirse con la verdadera escasez de especialistas enunciada antes y debe ser corregido simplemente a través de la gestión cotidiana de los establecimientos.

Cabe, entonces, ajustar las dotaciones de personal a los estándares en uso en el mercado y en los nuevos proyectos, de cara a las nuevas necesidades que surgen del desarrollo de la medicina y del ya cambiado perfil epidemiológico (envejecimiento poblacional).

Pero, por otra parte el Ministerio de Salud debe intervenir decididamente en el mercado del trabajo médico para aumentar la oferta de especialistas escasos, tanto en la formación en Chile y en el extranjero, cuanto como en incorporación directa de profesionales extranjeros.

Simultáneamente es necesario mejorar las remuneraciones de los médicos con foco en especialidades escasas, para cerrar las brechas con el sector privado que emergen de la escasez relativa. $\mathrm{Al}$ respecto, hacemos la hipótesis de que, en casos como el de los médicos que laboran en unidades de pacientes críticos, la productividad y carga de trabajo serían comparables entre sectores público y privado, pero es posible que éstas sean mayores en el sector público en atención a que sólo los casos más graves ocupan las escasas camas críticas, mientras en el sector privado se hospitalizan en dichas camas pacientes que en el sector público no las ocuparían.

La mejora señalada antes, sin embargo, debe ser muy cuidadosa y balanceada, pues en la medida que no se realice en paralelo un incremento de la oferta de especialistas, el resultado podría ser un escalamiento "simétrico" de las remuneraciones con el sector privado y un consiguiente aumento global de los costos para el sector, sin un correlato en mayor dotación de médicos disponibles para el sector público.

Por último, es necesario persistir en la selección de directivos hospitalarios a través del sistema de Alta Dirección Pública, incluyendo Dirección y Subdirecciones de hospitales EAR (hoy sólo se incluye la Subdirección Médica) y, por otra parte, ajuste a remuneraciones y riesgos de mercado.
Salvo el último punto (mejorar las rentas directivas superiores), las medidas que se proponen en materia de recursos humanos no deberían implicar más gastos para el sistema, pues son sólo transferencias de gasto entre el subtítulo 22 -donde vía compra de servicios ya se están pagando los precios de mercado y expandiendo las dotacionesal subtítulo 21, donde este gasto normalmente debería situarse.

\section{Referencias}

1. Comisión Presidencial de Trabajo para el Desarrollo de Propuesta de Financiamiento del Sector Salud y Licencias Médicas. Informe, 2010.

2. Comisión Técnica para el Diseño de un Plan Garantizado Universal para el sistema ISAPRE. Ministerio de Salud. Informe, 2011.

3. Vergara M. La Dicotomía Estado-Mercado del Sector de la Salud en Chile. Para Estudios Sociales. Corporación de Promoción Universitaria, Santiago, Chile. 2001; 108 (2): 115-31.

4. Harvard Business Review. La Toma de Decisiones. Recopilación de Drucker, P. y otros autores. Santiago de Chile, 2005.

5. Vergara M. ¿Qué hacer con las Isapre? Revista de la Facultad de Medicina, Número 7, Invierno 2014.

6. Enthoven, A. Health Plan: the practical solution for the soaring costs of medical care. Washington D.C. 2002.

7. Leavell HR, Clark F. Preventive Medicine for the Doctor in his Community. 1965.

8. Meyer J, Rowan B. Organizaciones Institucionalizadas: La Estructura Formal como Mito y Ceremonia. Capítulo I. Powell, W. \& Di Maggio, P. (compiladores) Serie Nuevas Lecturas de Política y Gobierno. Fondo de Cultura Económica. México. 1999.

9. Cunill Grau N, Fernández M, Vergara M. Gobernanza sistémica para un enfoque de derechos en salud. Un análisis a partir del caso chileno. Salud Colectiva 2011; 7: 21-3.

10. WHO (World Health Organization). The World Health Report 2000. Health Systems: Improving Performances. 2000.

11. Harvard Business Review. Porter, M. y Oldsberg, E. Redefiniendo la competencia en el sector salud. 2004.

12. Vergara R. Estudio Introductorio en March, J. \& Olsen, J. El Redescubrimiento de las Organizaciones: La Base Organizativa de la Política. Serie Nuevas Lecturas de Política y Gobierno. Fondo de Cultura Económica. México. 1997.

13. Castro R, Cid C, Díaz E, Carvajal J, Román A. “Meca- 
Reformas a prestadores públicos de servicios médicos - M. Vergara

nismos de Pago y capitación Ajustada por Riesgos en la Atención Sanitaria Pública Chilena”. OPS Chile. Economía y Salud: aportes y experiencias en América Latina 2011; 2: 139-57.

14. Escuela de Trabajo Social y Observatorio de Ciudades (PUC) y Expansiva. Gestión de Salud Pública y de provisión de servicios de salud. Para libro La Reforma Municipal en la Mira. 2007.
15. Vergara M, Bisama L, Moncada P. Competencias Esenciales para la Gestión en Red. Rev Med Chile 2012; 140: 1606-12.

16. OPS: Muñoz F, López-Acuña D, Halverson P, Guerra de Macedo C, Hanna W, Larrieu M, et al. Las funciones esenciales de la salud pública: un tema emergente en las reformas del sector de la salud. Rev Panam Salud Pública 2000; $8(1-2)$. 